\title{
A NOTE ON COMPLETE INTEGRALS
}

\author{
WOJCIECH CHOJNACKI
}

(Communicated by Barbara Lee Keyfitz)

\begin{abstract}
We present a theorem concerning the representation of solutions of a first-order partial differential equation in terms of a complete integral of the equation. We discuss the geometrical significance of that theorem.
\end{abstract}

\section{INTRODUCTION}

This note deals with the representation of solutions of a first-order partial differential equation in terms of complete integrals. A subject much discussed in many monographs on partial differential equations is generation of various classes of solutions by forming envelopes of families consisting of the graphs of solutions making up the complete integral. Less thoroughly studied is the question of whether, given a complete integral, the graph of any particular solution can be represented as an appropriate envelope. Addressing that issue, some authors point out difficulties in making general statements, while others make statements that contain flaws. This article attempts to fill gaps in the existing literature on the subject and to rectify erroneous assertions.

\section{REPRESENTATION OF SOLUTIONS}

Let $M$ be an open subset of $\mathbb{R}^{n}$, and, for each $k \in \mathbb{N}$, let $C^{k}(M)$ be the space of all real functions on $M$ that possess continuous partial derivatives of order $\leq k$. Given $u \in C^{1}(M)$, let $j_{0}(u): M \rightarrow M \times \mathbb{R}$ be the 0 -jet of $u$ over $M$ defined by

$$
\left[j_{0}(u)\right](x)=(x, u(x)) \quad(x \in M)
$$

and let $j_{1}(u): M \rightarrow M \times \mathbb{R} \times \mathbb{R}^{n}$ be the 1 -jet of $u$ over $M$ defined by

$$
\left[j_{1}(u)\right](x)=\left(x, u(x), \frac{\partial u}{\partial x_{1}}(x), \ldots, \frac{\partial u}{\partial x_{n}}(x)\right) \quad(x \in M) .
$$

Let $N$ be an open subset of $M \times \mathbb{R} \times \mathbb{R}^{n}$ and $F \in C^{1}(N)$. With $N$ and $F$ there is associated a first-order partial differential equation on $M$ defined as

Received by the editors April 27, 1993.

1991 Mathematics Subject Classification. Primary 35F99; Secondary 35A30, 53C42, 58A15, $58 \mathrm{~A} 20$.

Key words and phrases. First-order partial differential equation, complete integral, envelope. 
the set $E$ of those points $(x, u, p)$ in $N$ for which

$$
F(x, u, p)=0 .
$$

(This non-standard definition of a first-order partial differential equation appears natural from the standpoint of the theory of jet bundles (cf. [Lyc]). While our exposition will not resort to results of that theory, some notation and phrasings will allude to the possibility of ensconcing the material presented within the framework of jet bundles.) Let $\Omega$ be an open subset of $M$. A function $u \in C^{k}(\Omega)(k \in \mathbb{N})$ is said to be a $C^{k}$ solution of (1) on $\Omega$ if the image of $\Omega$ under $j_{1}(u)$ lies in $E$, that is, $\left[j_{1}(u)\right](\Omega) \subset E$. Let $\mathfrak{P}$ be an open subset of $\mathbb{R}^{n}$. A function $G \in C^{2}(M \times \mathfrak{P})$ is called a complete integral of (1) on $M \times \mathfrak{P}$ if

(Cli) for each $P \in \mathfrak{P}$, the function $G_{P}$ from $M$ into $\mathbb{R}$ given by

$$
G_{P}(x)=G(x, P) \quad(x \in M)
$$

is a $C^{2}$ solution of (1) on $M$;

(CIii) for each $x \in M$ and each $P \in \mathfrak{P}$, the rank of the matrix

$$
\left[\begin{array}{cccc}
\frac{\partial G}{\partial P_{1}} & \frac{\partial^{2} G}{\partial x_{1} \partial P_{1}} & \cdots & \frac{\partial^{2} G}{\partial x_{n} \partial P_{1}} \\
\vdots & \vdots & \ddots & \vdots \\
\frac{\partial G}{\partial P_{n}} & \frac{\partial^{2} G}{\partial x_{1} \partial P_{n}} & \cdots & \frac{\partial^{2} G}{\partial x_{n} \partial P_{n}}
\end{array}\right](x, P)
$$

is equal to $n$.

We have the following.

Theorem. With $M, N, F$, and $\mathfrak{P}$ as above, let $G$ be a complete integral of (1) on $M \times \mathfrak{P}$, and let $x^{0} \in M, u^{0} \in \mathbb{R}, p^{0} \in \mathbb{R}^{n}$, and $P^{0} \in \mathfrak{P}$ be such that

$$
\begin{gathered}
(\mathrm{d} F)\left(x^{0}, u^{0}, p^{0}\right) \neq 0, \\
{\left[j_{1}\left(G_{P 0}\right)\right]\left(x^{0}\right)=\left(x^{0}, u^{0}, p^{0}\right) .}
\end{gathered}
$$

If $u$ is a $C^{k}(k=1,2)$ solution to (1) on an open neighbourhood $\Omega \subset M$ of $x^{0}$ with

$$
\left[j_{1}(u)\right]\left(x^{0}\right)=\left(x^{0}, u^{0}, p^{0}\right),
$$

then there exists an open neighbourhood $U \subset \Omega$ of $x^{0}$ and a $C^{k-1}$ function $f$ from $U$ into $\mathfrak{P}$ such that

$$
f\left(x^{0}\right)=P^{0}
$$

and, for each $x \in U$,

$$
\left[j_{1}(u)\right](x)=\left[j_{1}\left(G_{f(x)}\right)\right](x) .
$$

Moreover, if, for some open neighbourhood $V \subset M$ of $x^{0}$, a continuous function $g$ from $V$ into $\mathfrak{P}$ satisfies (5) and (6), then $g$ and $f$ coincide on an open neighbourhood $V^{\prime} \subset V \cap U$ of $x^{0}$. 
Proof. Let $\varphi_{G}$ be the function from $M \times \mathfrak{P}$ into $E$ given by

$$
\varphi_{G}(x, P)=\left[j_{1}\left(G_{P}\right)\right](x) \quad(x \in M, P \in \mathfrak{P}),
$$

and let $\pi$ be the natural projection from $M \times \mathbb{R} \times \mathbb{R}^{n}$ onto $M$. We claim that there exist open neighbourhoods $W \subset M$ of $x^{0}, \mathscr{P} \subset \mathfrak{P}$ of $P^{0}$, and $\mathscr{U} \subset N$ of $\left(x^{0}, u^{0}, p^{0}\right)$ such that:

(i) $E \cap \mathscr{U}$ is a $2 n$-dimensional $C^{1}$ submanifold of $M \times \mathbb{R} \times \mathbb{R}^{n}$;

(ii) the restriction of $\varphi_{G}$ to $W \times \mathscr{P}$ is a $C^{1}$ diffeomorphism from $W \times \mathscr{P}$ onto $E \cap \mathscr{U}$.

Indeed, in view of (2), there exists an open subset $\mathscr{U}$ of $N$ containing $\left(x^{0}, u^{0}, p^{0}\right)$ such that (i) holds.

In view of (Clii), the mapping $\varphi_{G}$ has rank $2 n$ at all points in $M \times \mathfrak{P}$; that is, the differential $\left(\mathrm{d} \varphi_{G}\right)(x, P)$ of $\varphi_{G}(x, P)$ at every point $(x, P) \in M \times \mathfrak{P}$ has rank $2 n$. By the continuity of $\varphi_{G}$, there exist open neighbourhoods $W \subset$ $M$ of $x^{0}$ and $\mathscr{P} \subset \mathfrak{P}$ of $P^{0}$ such that $\varphi_{G}(W \times \mathscr{P})$ is contained in $E \cap \mathscr{U}$. Now (i) and the inverse function theorem ensure that, if necessary, one can shrink $W, \mathscr{P}$, and $\mathscr{U}$ so that (ii) holds. The claim is established.

Let $u$ be a $C^{k}(k=1,2)$ solution to (1) on an open neighbourhood $\Omega \subset M$ of $x^{0}$ satisfying (4). Then $j_{1}(u)$ is a mapping of class $C^{k-1}$, and $\left[j_{1}(u)\right](U) \subset$ $E \cap \mathscr{U}$ for some open neighbourhood $U \subset W \cap \Omega$ of $x^{0}$. Let $\eta$ be the natural projection from $M \times \mathfrak{P}$ onto $\mathfrak{P}$. Define a $C^{k-1}$ function $f$ from $U$ into $\mathfrak{P}$ by setting

$$
f(x)=\left(\eta \circ \varphi_{G}^{-1} \circ\left[j_{1}(u)\right]\right)(x) \quad(x \in U) .
$$

In view of (ii), this definition makes sense. It is easy to see that $f$ satisfies (5) and (6).

Suppose now that $g$ is a continuous function from an open neighbourhood $V$ of $x^{0}$ into $\mathfrak{P}$ satisfying (5) and (6). Then there exists an open neighbourhood $V^{\prime} \subset V \cap U$ of $x^{0}$ such that $g\left(V^{\prime}\right) \subset \mathscr{P}$. Since, for each $x \in V^{\prime}$, we have $(x, g(x)) \in W \times \mathscr{P},\left[j_{1}(u)\right](x) \in E \cap \mathscr{U}$, and $\varphi_{G}(x, g(x))=\left[j_{1}(u)\right](x)$, it follows from (ii) that, for each $x \in V^{\prime}, g(x)=\left(\eta \circ \varphi_{G}^{-1} \circ\left[j_{1}(u)\right]\right)(x)$, and hence $g(x)=f(x)$.

The above theorem permits reduction of the problem of finding $C^{2}$ solutions of (1) to that of solving a system of quasi-linear equations, namely, system (9) given below. In fact, if $u$ is a $C^{2}$ solution to (1) on an open subset $U \subset M$ such that (6) holds for some $C^{1}$ function $f$ from $U$ into $\mathfrak{P}$, then, for each $x \in U$,

$$
u(x)=G(x, f(x))
$$

and

$$
\frac{\partial u}{\partial x_{i}}(x)=\frac{\partial G}{\partial x_{i}}(x, f(x)) \quad(1 \leq i \leq n) .
$$

Differentiating (7) gives

$$
\frac{\partial u}{\partial x_{i}}(x)=\frac{\partial G}{\partial x_{i}}(x, f(x))+\sum_{j=1}^{n} \frac{\partial G}{\partial P_{j}}(x, f(x)) \frac{\partial f_{j}}{\partial x_{i}}(x) \quad(1 \leq i \leq n),
$$


which together with $(8)$ yields

$$
\sum_{j=1}^{n} \frac{\partial G}{\partial P_{j}}(x, f(x)) \frac{\partial f_{j}}{\partial x_{i}}(x)=0 \quad(1 \leq i \leq n) .
$$

In turn, if $f$ is a $C^{k}(k=1,2)$ function from $U$ into $\mathfrak{P}$ satisfying (9), then clearly $u$ given by (7) is a $C^{k}$ solution to (1). There are instances when (9) can easily be handled. For example, under the assumption that $f$ has constant rank at each point in $U$, (9) can be reduced to a system of algebraic equations (as we shall see shortly). Observe that for a given $C^{2}$ solution $u$, the corresponding function $f$ is, as a rule, merely of class $C^{1}$, whereas for a given $C^{1}$ function $f$, the solution given by (7) is of class $C^{1}$ but usually not of class $C^{2}$. Thus, in each particular case of employing (9) to solve (1), the question of differentiability of solutions requires a separate treatment.

We close this section by remarking that the conjunction of conditions (2), (3), and (4) constitutes a germ of the representation embodied by equations (5) and (6) of such a form that the difficulty in generating a result like the above theorem, mentioned in [CH, p. 25], can be overcome.

\section{ENVELOPES}

Let $\Omega$ be an open subset of $\mathbb{R}^{n}, A$ be a topological space, $\left\{v_{a}: a \in A\right\}$ be a family of functions in $C^{1}(\Omega)$ indexed by $A, w$ be a function in $C^{1}(\Omega)$, and $f$ be a continuous function from $\Omega$ onto $A$ such that $\left[j_{1}\left(v_{f(x)}\right)\right](x)=$ $\left[j_{1}(w)\right](x)$ for each $x \in \Omega$. Then the graph of $w$ over $\Omega$, $\left[j_{0}(w)\right](\Omega)$, is the envelope of the family of graphs $\left\{\left[j_{0}\left(v_{a}\right)\right](\Omega): a \in A\right\}$ with selector $f$. This means that given a point $(x, w(x))$ in $\left[j_{0}(w)\right](\Omega),(x, w(x))$ is also a member of $\left[j_{0}\left(v_{f(x)}\right)\right](\Omega)$, and, with both $\left[j_{0}(w)\right](\Omega)$ and $\left[j_{0}\left(v_{f(x)}\right)\right](\Omega)$ envisaged as subspaces of $\Omega \times \mathbb{R}$, the tangent spaces to $\left[j_{0}(w)\right](\Omega)$ and $\left[j_{0}\left(v_{f(x)}\right)\right](\Omega)$ at $(x, w(x))$ coincide.

Retaining the notation from the above theorem, suppose that $u$ is a $C^{2}$ solution to (1) on an open neighbourhood $U \subset M$ of $x^{0}$ such that (5) and (6) hold for some $C^{1}$ function $f$ from $U$ into $\mathfrak{P}$. Then, according to the previous paragraph, the graph $\left[j_{0}(u)\right](U)$ is the envelope of the family of graphs $\left\{\left[j_{0}\left(G_{P}\right)\right](U): P \in f(U)\right\}$ with selector $f$. If, for some open neighbourhood $V \subset U$ of $x^{0}$, the rank of $f$ at every point $x \in V$ is an integer $k$ independent of $x$ (so that $f(V)$ is a $k$-dimensional $C^{1}$ submanifold of $\mathfrak{P}$ ), then the graph $\left[j_{0}(u)\right](V)$ is the envelope of the $k$-parameter family $\left\{\left[j_{0}\left(G_{P}\right)\right](V): P \in f(V)\right\}$ with selector $f$, where the qualification " $k$-parameter" refers to the index set $f(V)$ being a $k$-dimensional $C^{1}$ submanifold of $\mathfrak{P}$. Assuming that $f$ has rank $k$ at every point in $V$, we now briefly analyse various cases that arise for different values of $k$. In doing so, we follow a pattern employed in many monographs on partial differential equations. For the sake of simplicity, we assume that $V$ coincides with $U$, that is, $f$ has rank $k$ at every point in $U$ itself.

Case 1. If $k=0$, then $f(x)=P^{0}$ and $u(x)=G\left(x, P^{0}\right)$ for all $x \in U$. Hence, the graph $\left[j_{0}(u)\right](U)$ is identical with the graph $\left[j_{0}\left(G_{P^{0}}\right)\right](U)$, and as 
such may be identified with the envelope of the 0-parameter family consisting of one single element $\left[j_{0}\left(G_{P^{0}}\right)\right](U)$.

Case 2. If $1 \leq k \leq n-1$, then, by shrinking $U$ if necessary, one can represent the graph $\left[j_{0}(u)\right](U)$ as an envelope of a $k$-parameter family of the form $\left\{\left[j_{0}\left(G_{R(a)}\right)\right](U): a \in A\right\}$, where $A$ is an open subset of $\mathbb{R}^{k}$ and $R(a \rightarrow R(a))$ is a $C^{1}$ function from $A$ into $\mathfrak{P}$. Indeed, let $I$ be a set of integers $i_{1}, \ldots, i_{k}$ with $1 \leq i_{1}<\cdots<i_{k} \leq n$ such that the function $g_{I}$ defined by

$$
g_{I}(x)=\left(f_{i_{1}}(x), \ldots, f_{i_{k}}(x)\right) \quad(x \in U)
$$

has rank $k$ at $x^{0}$, and let $J$ be the complement of $I$ in $\{1, \ldots, n\}$ consisting of integers $j_{1}, \ldots, j_{n-k}$ with $1 \leq j_{1}<\cdots<j_{n-k} \leq n$. An inspection of the standard proof to the rank theorem reveals that there exists an open neighbourhood $U^{\prime} \subset U$ of $x^{0}$ such that $g_{I}$ has rank $k$ at each point in $U^{\prime}$ (so that, in particular, $A=g_{I}\left(U^{\prime}\right)$ is an open subset of $\mathbb{R}^{k}$ ) and a $C^{1}$ function $\varphi_{J}=\left(\varphi_{j_{1}}, \ldots, \varphi_{j_{n-k}}\right)$ from $A$ into $\mathbb{R}^{n-k}$ such that, for each $x \in U^{\prime}$,

$$
f_{j}(x)=\varphi_{j}\left(g_{I}(x)\right) \quad(j \in J) .
$$

Let $R$ be a $C^{1}$ mapping from $A$ into $\mathfrak{P}$ given by

$$
R(a)_{k}=\left\{\begin{array}{ll}
a_{k} & \text { if } k \in I, \\
\varphi_{k}(a) & \text { if } k \in J
\end{array} \quad(a \in A) .\right.
$$

Since $g_{I}$ has rank $k$ at each point in $U^{\prime}$, it follows from (9) that, for each $x \in U^{\prime}$,

$$
\text { (11) } \frac{\partial G}{\partial P_{i}}\left(x, R\left(g_{I}(x)\right)\right)+\sum_{j \in J} \frac{\partial G}{\partial P_{j}}\left(x, R\left(g_{I}(x)\right)\right) \frac{\partial \varphi_{j}}{\partial a_{i}}\left(g_{I}(x)\right)=0 \quad(i \in I) \text {. }
$$

These equations immediately imply that the graph $\left[j_{0}(u)\right]\left(U^{\prime}\right)$ is the envelope of the family $\left\{\left[j_{0}\left(G_{R(a)}\right)\right]\left(U^{\prime}\right): a \in A\right\}$ with selector $g_{I}$. It is worth noticing that if $\varphi_{J}$ is assumed to be known, then (11) can be used to determine $g_{I}$ in a purely algebraic way (e.g., by employing the implicit function theorem), and next (8) and (10) can be used to generate a solution to (1). Details of that construction can be found in many monographs on partial differential equations (cf. [CH, §1.4.2; Dou, §2.6.1; Sne, §2.7]).

Case 3. If $k=n$, then $f(U)$ is an open subset of $\mathfrak{P}$ and the graph $\left[j_{0}(u)\right](U)$ is the envelope of the $n$-parameter family $\left\{\left[j_{0}\left(G_{P}\right)\right](U): P \in f(U)\right\}$ with selector $f$. Interestingly, in this case the function $f$ is determined in a unique fashion in the vicinity $x^{0}$, and so too is $u$. More precisely, we have the following.

Proposition 1. With $M, N, F$, and $\mathfrak{P}$ as above, let $G$ be a complete integral of (1) on $M \times \mathfrak{P}, x^{0} \in M, U \subset M$ be an open neighbourhood of $x^{0}, P^{0} \in \mathfrak{P}$, and $f$ be a $C^{1}$ function from $U$ into $\mathfrak{P}$ satisfying (5) and (9), and having rank $n$ at each point in $U$. If, for some open neighbourhood $V \subset M$ of $x^{0}, a$ $C^{1}$ function $g$ from $V$ into $\mathfrak{P}$ satisfies (5) and (9), and has rank $n$ at each point in $V$, then $g$ and $f$ coincide on some open neighbourhood $V^{\prime} \subset V \cap U$ of $x^{0}$. 
Proof. For each $x \in U,(9)$ and the fact that $f$ has rank $n$ at $x$ imply that

$$
\frac{\partial G}{\partial P_{j}}(x, f(x))=0 \quad(1 \leq j \leq n) .
$$

Hence, in view of (CIii),

$$
\left|\begin{array}{ccc}
\frac{\partial^{2} G}{\partial x_{1} \partial P_{1}} & \cdots & \frac{\partial^{2} G}{\partial x_{n} \partial P_{1}} \\
\vdots & \ddots & \vdots \\
\frac{\partial^{2} G}{\partial x_{1} \partial P_{n}} & \cdots & \frac{\partial^{2} G}{\partial x_{n} \partial P_{n}}
\end{array}\right|(x, f(x)) \neq 0 .
$$

Differentiating (12), we obtain

$$
\begin{aligned}
& \frac{\partial^{2} G}{\partial x_{i} \partial P_{j}}(x, f(x))+\sum_{k=1}^{n} \frac{\partial^{2} G}{\partial P_{j} \partial P_{k}}(x, f(x)) \frac{\partial f_{k}}{\partial x_{i}}(x)=0 \\
&(1 \leq i \leq n, \quad 1 \leq j \leq n) .
\end{aligned}
$$

This together with (13) shows that

$$
\left|\begin{array}{ccc}
\frac{\partial^{2} G}{\partial P_{1} \partial P_{1}} & \cdots & \frac{\partial^{2} G}{\partial P_{n} \partial P_{1}} \\
\vdots & \ddots & \vdots \\
\frac{\partial^{2} G}{\partial P_{1} \partial P_{n}} & \cdots & \frac{\partial^{2} G}{\partial P_{n} \partial P_{n}}
\end{array}\right|(x, f(x)) \neq 0 .
$$

Now (5), (12), and (15) imply that

$$
\frac{\partial G}{\partial P_{j}}\left(x^{0}, P^{0}\right)=0 \quad(1 \leq j \leq n)
$$

and

$$
\left|\begin{array}{ccc}
\frac{\partial^{2} G}{\partial P_{1} \partial P_{1}} & \cdots & \frac{\partial^{2} G}{\partial P_{n} \partial P_{1}} \\
\vdots & \ddots & \vdots \\
\frac{\partial^{2} G}{\partial P_{1} \partial P_{n}} & \cdots & \frac{\partial^{2} G}{\partial P_{n} \partial P_{n}}
\end{array}\right|\left(x^{0}, P^{0}\right) \neq 0
$$

Suppose that for some open neighbourhood $V \subset M$ of $x^{0}$, a $C^{1}$ function $g$ from $V$ into $\mathfrak{P}$ satisfies (5) and (9), and has rank $n$ at each point in $V$. Then $g$ also satisfies (12). The existence of an open neighbourhood $V^{\prime} \subset V \cap U$ of $x^{0}$ such that $g$ and $f$ coincide on $V^{\prime}$ now follows from (16) and (17) upon applying (the uniqueness part of) the implicit function theorem.

Any $C^{2}$ function $u$ given by (5), where $G$ is a complete integral of (1) and $f$ is a $C^{1}$ function from an open set $U \subset M$ into $\mathfrak{P}$ satisfying (9) and having rank $n$ at each point in $U$, is called a singular solution to (1). It is easy to see that every singular solution $u$ satisfies, along with (1), the system of equations

$$
\frac{\partial F}{\partial p_{i}}(x, u, p)=0 \quad(1 \leq i \leq n) .
$$

In fact, differentiating the relation

$$
F \circ\left(\left[j_{1}\left(G_{P}\right)\right](x)\right)=0
$$


yields

$$
\begin{aligned}
\frac{\partial F}{\partial u}\left(\left[j_{1}\left(G_{P}\right)\right](x)\right) \frac{\partial G}{\partial P_{j}}(x, P)+\sum_{i=1}^{n} \frac{\partial F}{\partial p_{i}}\left(\left[j_{1}\left(G_{P}\right)\right](x)\right) \frac{\partial^{2} G}{\partial x_{i} \partial P_{j}}(x, P) & =0 \\
& (1 \leq j \leq n) .
\end{aligned}
$$

Using the latter identity together with (6), (12), and (13), we readily find that $u$ indeed satisfies (18). The system consisting of (1) and all of the equations (18) can be used to obtain a characterisation of singular solutions that makes no appeal to complete integrals (cf. [CH, §1.4.3; Dou, Assertion 1.6.3]).

\section{AN EXAMPLE}

It may happen that, for any open neighbourhood $U$ of $x^{0}$, the rank of $f$ at a point $x \in U$ varies as $x$ runs over $U$, and so $f(U)$ is not a $C^{1}$ submanifold of $\mathfrak{P}$. Below we present an example elucidating this possibility. Not taking into account that, for any open neighbourhood $U$ of $x^{0}, f(U)$ may fail to be a $C^{1}$ submanifold of $\mathfrak{P}$ has resulted in a flaw in a statement in [Sne, p. 61] specifying all possible types of envelopes with which to represent the graph of a given solution. (According to that invalid statement, every envelope falls into one of the three categories discussed in the previous section.)

Our example will reveal a situation in which, given an integer $k$ with $1 \leq$ $k \leq n-1$, for any open neighbourhood $U$ of $x^{0}$ the rank of $f$ is equal to $\bar{k}$ at each point in $U \backslash\left\{x^{0}\right\}$ and drops to $k-1$ at $x^{0}$.

Example. Let $n$ be an integer with $n \geq 2, M=\left\{\left(x_{1}, x_{2}, \ldots, x_{n}\right) \in \mathbb{R}^{n}: x_{2} \neq\right.$ $0\}, \mathfrak{P}=\mathbb{R}^{n}$, and

$$
F(x, u, p)=\left\{\begin{array}{ll}
\sum_{i=1}^{n} x_{i} p_{i}-\frac{1}{2} \sum_{i=3}^{n} p_{i}^{2}-u & \text { if } n \geq 3, \\
x_{1} p_{1}+x_{2} p_{2}-u & \text { if } n=2
\end{array} \quad\left(x \in M, u \in \mathbb{R}, p \in \mathbb{R}^{n}\right) .\right.
$$

It is easy to see that the function $G$ given by

$$
G(x, P)=\left\{\begin{array}{ll}
\sum_{i=1}^{n} x_{i} P_{i}-\frac{1}{2} \sum_{i=3}^{n} P_{i}^{2} & \text { if } n \geq 3, \\
x_{1} P_{1}+x_{2} P_{2} & \text { if } n=2
\end{array} \quad(x \in M, P \in \mathfrak{P})\right.
$$

is a complete integral of (1), with $F$ given by (19), on $M \times \mathfrak{P}$. Let $k$ be an integer such that $1 \leq k \leq n-1, x^{0} \in M$ be such that $x_{1}^{0}=0$,

$$
u^{0}= \begin{cases}\frac{1}{2} \sum_{i=3}^{k+1}\left(x_{i}^{0}\right)^{2} & \text { if } k \geq 2 \\ 0 & \text { if } k=1\end{cases}
$$

$p^{0}$ and $P^{0}$ be such that $p_{i}^{0}=P_{i}^{0}=0$ for $i \in\{1,2\} \cup\{j \in \mathbb{N}: k+2 \leq j \leq n\}$, and $p_{i}^{0}=P_{i}^{0}=x_{i}^{0}$ for $3 \leq i \leq k+1$. Then, clearly, (2) and (3) are satisfied. 
Moreover, the function $u$ given by

$$
u(x)=\left\{\begin{array}{ll}
x_{1}^{3} x_{2}^{-2}+\frac{1}{2} \sum_{i=3}^{k+1} x_{i}^{2} & \text { if } k \geq 2, \\
x_{1}^{3} x_{2}^{-2} & \text { if } k=1
\end{array} \quad(x \in M)\right.
$$

is a $C^{2}$ solution to (1), with $F$ given by (19), on $M$ satisfying (4). Let $U \subset M$ be an open neighbourhood of $x^{0}$, and let $f=\left(f_{1}, \ldots, f_{n}\right)$ be a $C^{1}$ function from $U$ into $\mathfrak{P}$ satisfying (6). Since, for each $x \in U$,

$$
\frac{\partial G}{\partial x_{i}}(x, f(x))=f_{i}(x) \quad(1 \leq i \leq n),
$$

it follows from (8) that

$$
\begin{array}{ll}
f_{1}(x)=3 x_{1}^{2} x_{2}^{-2}, & f_{2}(x)=-2 x_{1}^{3} x_{2}^{-3} \\
f_{i}(x)=x_{i} \text { if } 3 \leq i \leq k+1, & f_{i}(x)=0 \text { if } k+2 \leq i \leq n .
\end{array}
$$

It is easy to see that the rank of $f$ is equal to $k$ at every point in $U \backslash\left\{x^{0}\right\}$ and is equal to $k-1$ at $x^{0}$. Moreover, it is clear that all the intersections of $f(U)$ with sufficiently small open disks centered at $P^{0}$ take the form

$$
\begin{aligned}
& \left\{\left(3 t_{1}^{2},-2 t_{1}^{3}, t_{2}, \ldots, t_{k}, 0, \ldots, 0\right) \in \mathbb{R}^{n}:\right. \\
& \left.t_{1}^{4}\left(4 t_{1}^{2}+9\right)+\left(t_{2}-x_{3}^{0}\right)^{2}+\cdots+\left(t_{k}-x_{k+1}^{0}\right)^{2}<\delta\right\}
\end{aligned}
$$

if $k \geq 2$ and $\left\{\left(3 t^{2},-2 t^{3}\right):-\delta<t<\delta\right\}$ if $k=1$ for suitable positive $\delta$ 's. Thus, when $k \geq 2, f(U)$ has a cuspidal edge at points of the form $\left(0,0, t_{2}, \ldots, t_{k}, 0, \ldots, 0\right)$ and, when $k=1, f(U)$ has a cusp at $P^{0}$. Consequently, $f(U)$ is not a $C^{1}$ submanifold of $\mathfrak{P}$ in the vicinity of $P^{0}$.

We close this section by showing that the above example cannot be extended to $k=n$ for a fundamental reason.

Proposition 2. With $M, N, F$, and $\mathfrak{P}$ as above, let $G$ be a complete integral of (1) on $M \times \mathfrak{P}, U$ be an open connected subset of $M$, and $f$ be a $C^{1}$ function from $U$ into $\mathfrak{P}$ satisfying (9) and having rank $n$ at some point in $U$. Then $f$ has rank $n$ at each point in $U$.

Proof. Let $D$ be the set of those points in $U$ at which $f$ has rank $n$. Clearly, $D$ is an open non-empty subset of $U$. Repeating the argument from the proof of Proposition 1, we see that (12) and (14) hold for every point in $D$. Let $\bar{D}$ denote the closure of $D$ in $U$. By the continuity of $f$ and the first-order derivatives of $G,(12)$ is also valid for every point in $\bar{D}$. Similarly, the continuity of $f$ and the second-order derivatives of $G$ ensure the validity of (14) for all points in $\bar{D}$. Now, comparison of (12) and (Clii) shows that (13) holds for every point in $\bar{D}$. Furthermore, (13) and (14) imply that $f$ has rank $n$ at each point in $\bar{D}$. Thus $\bar{D} \subset D$ and further $D=\bar{D}$, showing that $D$ is a closed subset of $U$. Finally, by the connectedness of $U$, we have $D=U$, and the proposition follows. 


\section{REFERENCES}

[CH] R. Courant and D. Hilbert, Methods of mathematical physics, Vol. 2, Interscience, New York, 1962.

[Dou] A. Dou, Lectures on partial differential equations of first order, Univ. of Notre Dame Press, Notre Dame, IN, 1972.

[Lyc] V. V. Lychagin, Local classification of non-linear first order partial differential equations, Uspekhi Mat. Nauk 30 (1975), no. 1, 101-171; English transl. in Russian Math. Surveys (1) 30 (1975).

[Sne] I. Sneddon, Elements of partial differential equations, McGraw-Hill, New York, 1957.

INStytut Matematyki Stosowanej i Mechaniki, Uniwersytet Warszawski, Ul. Banacha 2, 02-097 Warszawa, Poland

E-mail address: wojtekch@appli.mimuw.edu.pl

Department of Computer Science, University of Adelaide, Adelaide, South Australia 5005, Australia

E-mail address: wojtek@cs.adelaide.edu.au 